\title{
Modelling of Flexible Manipulator System Using Flower Pollination Algorithm
}

\author{
Fadhli Muiz Talib \\ Faculty of Mechanical Engineering \\ Universiti Teknologi MARA \\ Shah Alam, Malaysia \\ fadhlimuiz@gmail.com \\ Annisa Jamali \\ Faculty of Engineering \\ Universiti Malaysia Sarawak \\ Kota Samarahan, Malaysia \\ jannisa@unimas.my
}

\author{
Muhamad Sukri Hadi \\ Faculty of Mechanical Engineering \\ Universiti Teknologi MARA \\ Shah Alam, Malaysia \\ msukrihadi@uitm.edu.my \\ Mat Hussin Ab Talib \\ School of Mechanical Engineering \\ Universiti Teknologi Malaysia \\ Johor, Malaysia \\ mathussin@utm.my
}

\author{
Hanim Mohd Yatim \\ School of Mechanical Engineering \\ Universiti Teknologi Malaysia \\ Johor, Malaysia \\ hanim.my@utm.my \\ Intan Zaurah Mat Darus \\ School of Mechanical Engineering \\ Universiti Teknologi Malaysia \\ Johor, Malaysia \\ intan@utm.my
}

\begin{abstract}
The study of the flexible manipulator system (FMS) has attracted many researchers due to its superiority of light weight and faster system response. Flexible manipulator system is an improvement from its rigid structure, however it can be easily vibrated when it subjected to disturbance. If the advantages of FMS are not to be sacrificed, an accurate model and efficient control system must be developed. Thus, this study presents an approach of evolutionary swarm algorithm via flower pollination algorithm (FPA) to model the dynamic system of flexible manipulator structure. An experimental rig of flexible manipulator system was developed for input-output acquisition. Then, this input-output data was fed to system identification method to obtain a dynamic model of flexible manipulator system utilizing evolutionary algorithm with linear auto regressive with exogenous (ARX) model structure. The result obtained through flower pollination algorithm was then compared with conventional method known as least square (LS) algorithm in terms of mean square error (MSE), correlation test and pole-zero diagram. The best MSE achieved by LS modeling for endpoint acceleration and hub angle positioning are 0.0075 and 0.0028 , respectively. While, the best MSE produced by flower pollination algorithm for endpoint acceleration and hub angle positioning are 0.0063 and 0.0020 , respectively. It is reveals that the performance of intelligence algorithm is superior than conventional algorithm.
\end{abstract}

Keywords-Flower pollination algorithm, evolutionary swarm algorithm, flexible manipulator, system identification

\section{INTRODUCTION}

Nowadays, flexible manipulators have been employed in many industries especially in automation and manufacturing fields, spacecraft and aircraft engineering which required that the weight of mechanical structures to be kept as low as possible. Flexible manipulators are designed to increase the productivity through its faster system response and higher manipulation speed while simultaneously consume lower energy due to its lighter weight [1]. Therefore, flexible manipulator systems have received substantial attention in recent years and being utilized in more complex tasks such as assembling and working at unmanned places [2].
However, the flexibility of the flexible manipulators can be easily affected by vibration when subjected to disturbance forces due to its low stiffness. The vibration will occur during maneuver and become more severe especially at higher speed motion [3]. This unwanted vibration will reduce the accuracy during positioning of flexible manipulator and thus its effectiveness. Thus, it is crucial to model and control this non-linear dynamic of the system.

This study represents part of the continuous effort in finding accurate dynamic model with intelligent optimization technique associating real input output data from experimental study in improving flexible manipulator system. Many researches had used system identification technique to developed variety of models. The system identification will establish a mathematical model and estimate model parameters that can represent the behaviour of the system based on input-output of the system [4]. Several researchers still applied this method since it is well developed and widely used to solve parametric modelling problems $[5,6]$.

Swarm algorithm are among of the evolutionary algorithm group that have been successfully used to compute the parameter values in any difficult applications. One from this class is flower pollination algorithm (FPA). FPA is a newly developed heuristic optimization method based on pollination of flowers. Yang investigate FPA on benchmark functions. Results showed that the performance of FPA outperforms Genetic Algorithm (GA) and Particle Swarm Optimization (PSO) algorithm [7]. Emary and his friends presents FPA for the optimization of retinal vessel segmentation. Results indicated that FPA has very fast convergence and robust even with abnormal images [8]. FPA has better quality solution and robustness because it has capabilities such as extensive domain search with quality, consistency solution and its performance encourage to implement for present problem [9]. 